Reprod. Nutr. Dévelop., 1987, 27 (2 B), 481-489.

\title{
Caractérisation de l'effet stimulant de deux neuro-peptides (thyréolibérine - TRH - et peptide vaso-actif intestinal - VIP -) sur la sécrétion de l'hormone de croissance (GH) du rat
}

\author{
D. DURAND $\left({ }^{1}\right)$, Marie-Thérèse BLUET-PAJOT $\left({ }^{*}\right)$, Françoise MOUNIER $\left({ }^{*}\right)$, \\ J. F. LEONARD (*), Monique PRESSAC
}

Laboratoire d'Endocrinologie, Faculté de Pharmacie, 4 avenue de l'Observatoire, 75270 Paris Cedex 06.

(*) INSERM, Unité de Neuroendocrinologie, U 159, 2 ter rue d'Alésia, 75014 Paris.

Summary. Further evidence for a stimulatory effect of two neuropeptides (thyrotropinreleasing hormone and vasoactive intestinal peptide) on growth hormone secretion in the rat.

The effects of thyrotropin-releasing hormone (TRH) and vasoactive intestinal peptide (VIP) on growth hormone (GH) secretion were investigated in vivo in freely moving, intact or mediobasal hypothalamic-lesioned rats and in vitro in incubation or superfusion systems of anterior pituitary tissue. In vivo, the two peptides induced a rapid increase of plasma GH levels in freely moving animals, bearing an extensive lesion of the mediobasal hypothalamus including the median eminence. VIP, but not TRH, was also effective in intact animals. In vitro, TRH and VIP stimulated GH release from incubated or superfused rat pituitaries in a concentrated manner : apparent affinity was $1.22 .10^{-8} \mathrm{M}$ for TRH and $2.5 .10^{-9} \mathrm{M}$ for VIP. These findings indicate that TRH and VIP exert a direct stimulatory action on somatotrophs, but that the effect of TRH (not observed in freely moving, intact rats) may be modulated by still other unidentified hypothalamic neurohormones.

\section{Introduction.}

Les sécrétions antéhypophysaires subissent un contrôle hypothalamique impliquant de nombreux facteurs. Ainsi, si deux facteurs hypothalamiques, la somatolibérine (GRF) (Guillemin et al., 1982) et la somatostatine (SRIF) (Brazeau et al., 1973) jouent un rôle majeur dans la régulation de la sécrétion somatotrope, il apparaît de plus en plus évident que d'autres neurohormones participent à cette régulation. Parmi ces neurohormones, deux peptides, la thyréolibérine (TRH) et le peptide vasoactif intestinal (VIP), pourraient au regard de la littérature exercer une action sur la sécrétion de GH. Toutefois, leurs effets décrits sont souvent contradictoires.

(1) Correspondance à adresser à : D. Durand, Laboratoire d'Endocrinologie, Faculté de Pharmacie, 4 avenue de l'Observatoire, 75270 Paris Cedex 06. 
La TRH, sans effet chez l'homme normal, stimule la sécrétion de GH au cours de différents états pathologiques et en particulier dans l'acromégalie (Irie et Tsushima, 1972). Chez le rat, le tripeptide stimule (Chihara et al., 1976a ; Kato et al., 1975), inhibe (Brown et Vale, 1975 ; Taché et al., 1977) ou ne modifie pas (Müller et al., 1977) la sécrétion somatotrope selon les auteurs. De semblables contradictions se retrouvent dans les expériences réalisées in vitro (Adams, Brajkovich et Mashiter, 1981, Borges et al., 1983 ; Carlson, Maritz et Daughaday, 1974 ; Szabo et al., 1984).

Les effets du VIP sur la sécrétion somatotrope sont également controversés. La stimulation de la sécrétion de GH par le VIP ne se manifeste chez le rat que si le peptide est administré dans le $3^{\mathrm{e}}$ ventricule et non par voie intraveineuse (Vijayan et al., 1979), ce qui suggère un site d'action suprahypophysaire ; hypothèse étayée par l'absence in vitro de libération de $\mathrm{GH}$ par des hypophyses (Enjalbert et al., 1980 ; Vijayan et al., 1979) ou des cellules hypophysaires (Rotsztejn et al., 1980 ; Vigh et Schally, 1984) après addition de VIP au milieu d'incubation ou de périfusion. Toutefois, des réponses somatotropes au VIP ont été rapportées aussi bien sur des cellules tumorales humaines (Chihara et al., 1982 ; Matsushita et al., 1981 ; White et al., 1985) ou murines (Aizawa et Hinkle, 1985 ; Dorflinger et Schonbrunn, 1983) que sur des cultures de cellules hypophysaires de rat après traitement par la dexaméthasone (Denef, Schramme et Baes, 1985).

Devant ces résultats dont la contradiction s'explique en partie par la diversité des conditions expérimentales, nous avons tenté de préciser le rôle de la TRH et du VIP sur la sécrétion somatotrope en utilisant différents modèles in vivo (rat non anesthésié intact ou porteur d'une lésion de I'hypothalamus médiobasal) et in vitro (incubation et périfusion d'antéhypophyses).

\section{Matériel et méthodes.}

Animaux. - Les expériences sont réalisées sur des rats mâles Wistar (Evic Ceba, Blanquefort 33 Francel pesant 250 à $300 \mathrm{~g}$, maintenus pendant au moins 2 semaines dans une animalerie climatisée $\left(22^{\circ} \pm 1^{\circ} \mathrm{C}\right)$ et éclairée de $7 \mathrm{~h}$ à $19 \mathrm{~h}$ ou de $2 \mathrm{~h}$ à $14 \mathrm{~h}$ selon les expériences.

Lésions. - La destruction de l'hypothalamus médiobasal et la section de la tige pituitaire sont obtenues selon la technique décrite par Dunn et Arimura (1974) à I'aide d'un couteau de type Halasz ayant la forme d'un triangle de $3,5 \mathrm{~mm}$ de côté. Les animaux lésés sont utilisés 4 jours après l'opération. La lésion présente la forme d'un triangle dont la base se situe au niveau de l'éminence médiane et le sommet à proximité des noyaux paraventriculaires. Se trouvent ainsi détruits les noyaux périventriculaires, arqués et ventromédians, une partie de l'hypothalamus basolatéral et l'éminence médiane.

Etudes in vivo. - Ces études sont réalisées chez des animaux non anesthésiés pouvant se déplacer librement dans leur cage. Pour cela avant chaque expérience un cathéter en silastic (Dow Corning) est introduit par la veine jugulaire externe droite jusqu'à I'oreillette droite. 
L'extrémité libre du cathéter est connectée à un tube en polyéthylène (ClayAdams) ressortant au niveau de la nuque. Ce dispositif permet les prélèvements de sang et l'injection soit des produits étudiés, soit d'hématies mises en suspension dans une solution de $\mathrm{NaCl} 150 \mathrm{mmol} / \mathrm{I}$ pour limiter au minimum les modifications hémodynamiques que pourraient entraîner les prélèvements répétés. Seuls les animaux ayant récupéré leur poids préopératoire sont utilisés 4 jours après la mise en place du cathéter.

\section{Etudes in vitro.}

a) Incubation. Après décapitation des animaux, l'anté-hypophyse est prélevée et sectionnée longitudinalement. Les demi-hypophyses obtenues sont incubées dans $1 \mathrm{ml}$ de milieu 199-Hépès (Gibco) maintenu à $37^{\circ} \mathrm{C}$ et saturé avec un mélange $\mathrm{O}_{2}-\mathrm{CO}_{2}(95 \%-5 \%)$. Après 2 préincubations d'une heure, les demihypophyses sont incubées $20 \mathrm{~min}$ en présence des différentes substances étudiées (pour chaque hypophyse une moitié sert de témoin à l'autre moitié). A la fin de l'incubation, le milieu est recueilli et congelé jusqu'au dosage. Les demihypophyses placées dans $1 \mathrm{ml}$ de $\mathrm{NaHCO}_{3} 0,05 \mathrm{M}, \mathrm{pH} 10$ sont détruites par les ultra-sons puis centrifugées à $3000 \mathrm{~g}$ pendant $20 \mathrm{~min}$. Le surnageant est congelé jusqu'au dosage.

b) Périfusion. - L'hypophyse, prélevée après décapitation des animaux, est coupée en 8 fragments, lavée pendant $30 \mathrm{~min}$ dans le milieu 199-Hépès (Gibco) puis placée dans une chambre de périfusion de $300 \mu \mathrm{l}$ maintenue à $37^{\circ} \mathrm{C}$ où elle sera lavée avec le même milieu à un débit de $100 \mu \mathrm{l} / \mathrm{min}$ pendant $120 \mathrm{~min}$, c'est-àdire jusqu'à l'obtention d'une sécrétion basale de GH stable. Ensuite, les substances étudiées sont ajoutées au milieu et l'effluent est recueilli toutes les 1 ou $3 \mathrm{~min}$ avant d'être congelé jusqu'au dosage. Les résultats sont exprimés en $\mathrm{ng} / \mathrm{min}$ et en $|\triangle|$, en ng d'hormone libérée. $|\Delta|$ représente la quantité totale d'hormone libérée pendant la réponse secrétoire à la substance étudiée, diminuée de la quantité d'hormone libérée pendant la même période dans les conditions basales.

Produits. - La TRH a été gracieusement fournie par Roche (Neuilly, 92 France). Le VIP provient de Sigma Chemical Company.

Dosage de l'hormone de croissance. - Les concentrations de GH sont déterminées par dosage radio-immunologique utilisant les réactifs fournis par le NIADDK (Md, USA) contre les standards $\mathrm{GH}-\mathrm{RP}_{1}$ et $\mathrm{GH}-\mathrm{RP}_{2}$.

Analyse statistique. - Les résultats représentent la moyenne \pm SEM. La comparaison des groupes est réalisée à l'aide du test $t$ de Student ou du test $U$ de Mann et Whitney selon les cas.

\section{Résultats.}

Expériences " in vivo ».

La figure 1 montre que l'injection intraveineuse de doses croissantes de TRH n'a pas d'effet sur les concentrations plasmatiques de GH de l'animal intact. En revanche, la TRH entraîne une augmentation significative et dépendant de la dose 
des concentrations plasmatiques de $\mathrm{GH}$ des animaux présentant une lésion importante de l'hypothalamus. L'effet de la TRH, qui se prolonge 20 à 30 min selon la dose, atteint son maximum 10 min après l'injection.

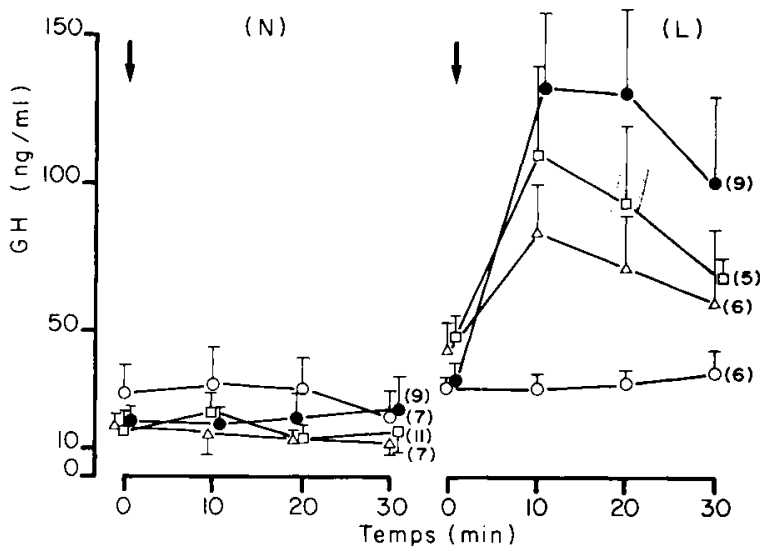

FIG. 1. - Etude de l'effet d'une injection de $\mathrm{NaCl} 150 \mathrm{mmol/l}(1)$ ou de $0,4(\mathrm{C}), 2(\square)$ ou $10 \mu \mathrm{g} / 100 \mathrm{~g}(\bullet)$ de TRH sur les concentrations plasmatiques de $\mathrm{GH}$ de rats vigiles intacts (N) ou lésés (L). Le nombre d'animaux utilisés est indiqué entre parenthèses.

Le VIP, à la dose de $2,5 \mu \mathrm{g} / 100 \mathrm{~g}$ provoque (fig. 2) une élévation significative de GH tant chez l'animal normal que chez l'animal lésé. La réponse atteint son maximum dans les $5 \mathrm{~min}$ qui suivent l'injection et se prolonge pendant $20 \mathrm{~min}$.

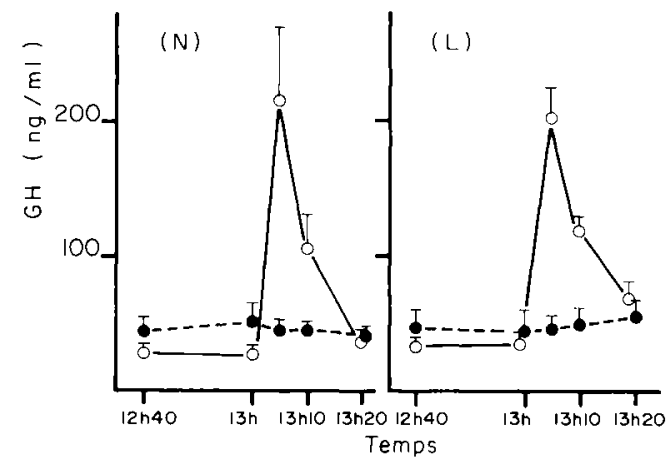

FIG. 2. - Etude de l'effet d'une injection de $\mathrm{NaCl} 150 \mathrm{mmol} / \mathrm{l}(\bullet)$ ou de $2,5 \mu \mathrm{g} / 100 \mathrm{~g}$ de VIP/) sur les concentrations plasmatiques de $\mathrm{GH}$ de rats vigiles intacts $(\mathrm{N})$ ou lésés (L). Le nombre d'animaux utilisés est indiqué entre parenthèses.

\section{Expérience " in vitro ».}

La TRH à la dose de $10^{-7} \mathrm{M}$ provoque la libération de $\mathrm{GH}$ par des hypophyses en périfusion (fig. 3). La moyenne des $|\triangle|$ est de $105 \pm 9,9 \mathrm{ng}$. L'augmentation est rapide et de courte durée $(30 \mathrm{~min})$. Des doses croissantes de TRH (fig. 4) induisent une stimulation dose dépendante de la libération de $\mathrm{GH}$ par des demi- 
hypophyses provenant aussi bien de rats intacts que de rats lésés. Pour cet effet, la TRH présente une affinité apparente (calculée selon Parker et Waud, 1971) de $1,22 \pm 0,4 \times 10^{-8} \mathrm{M}$.

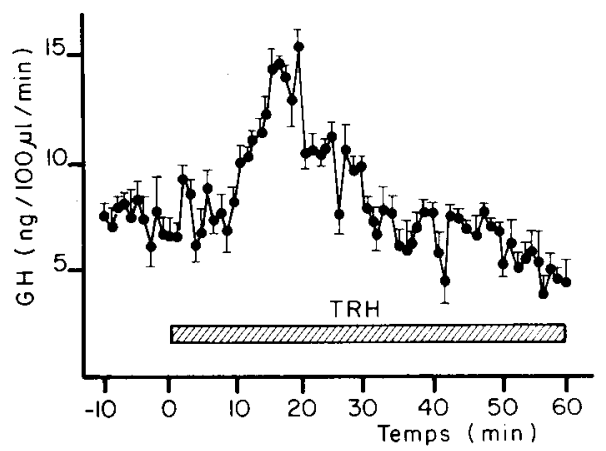

FIG. 3. - Effet de la TRH $\left(10^{-7} \mathrm{M}\right)$ sur la libération de $\mathrm{GH}$ par des hypophyses périfusées $(\mathrm{n}=4)$.

FIG. 4. - Effet des doses croissantes de TRH sur la sécrétion de GH lors d'incubation de demi-hypophyses provenant de rats intacts (N) ou lésés (L). Les résultats sont exprimés en \% de stimulation de la sécrétion de $\mathrm{GH}$ par rapport aux demi-hypophyses témoins.

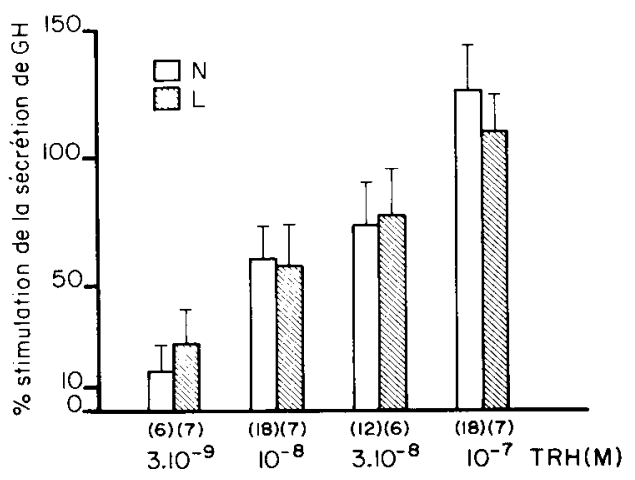

Le VIP, à des concentrations croissantes (fig. 5), stimule la libération de GH par des demi-hypophyses incubées avec une affinité apparente (calculée selon Parker et Waud, 1971) de $2,5 \times 10^{-9} \mathrm{M}$.

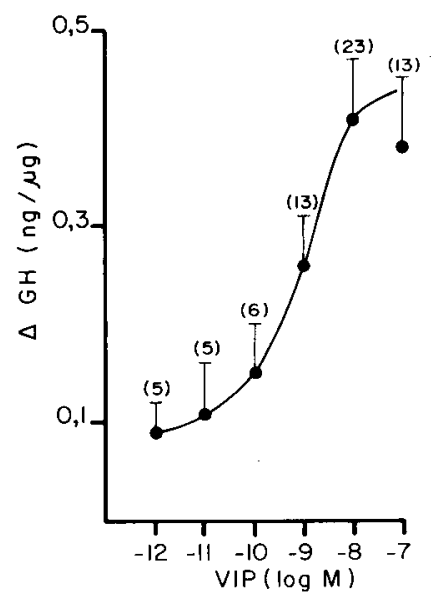

FIG. 5. - Effet de doses croissantes de VIP sur la libération de GH au cours de l'incubation de demi-hypophyses. Les résultats représentent la différence entre les rapports du contenu du milieu plus tissu $(M+T)$ des demi-hypophyses traitées (T) et des contrôles (C) correspondants:

$$
\frac{M_{T}}{M_{T}+T_{T}}-\frac{M_{C}}{M_{C}+T_{C}}
$$

Reproduction, Nutrition, Développement $n^{\circ} 2$ B-1987. -4 . 


\section{Discussion.}

Cette étude confirme la complexité de la réponse somatotrope à la TRH qui contraste avec la réponse au VIP. La TRH ne modifie pas la concentration plasmatique de $\mathrm{GH}$ de l'animal éveillé intact mais l'élève de façon dose-dépendante chez l'animal porteur d'une lésion de l'hypothalamus médiobasal. Cette augmentation évoque les résultats obtenus chez le rat après large destruction de l'hypothalamus (Chihara et al., 1976a) ou lésion électrolytique de l'éminence médiane (Müller et al., 1977). Toutefois, dans ces études, la relation dose-effet n'est pas clairement établie. Une stimulation de la sécrétion somatotrope par la TRH est également obtenue chez l'animal anesthésié à I'hydrate de chloral (Bluet-Pajot et al., 1986), I'uréthane (Kato et al., 1975) ou l'éther (Chihara et al., 1976a). Mais c'est une inhibition de la sécrétion de GH qui est observée par administration de TRH à l'animal anesthésié au pentobarbital (Brown et Vale, 1975 ; Taché et al., 1977). Ainsi, les anesthésiques affectent non seulement la sécrétion de GH (Kato et al., 1975 ; Martin, 1973-1974) mais également la réponse sécrétoire de GH à différents stimulus. II apparaît que la sensibilité de la sécrétion somatotrope aux anesthésiques rend contestable l'interprétation des expériences effectuées chez l'animal anesthésié.

Le VIP élève les concentrations plasmatiques de $\mathrm{GH}$ de l'animal normal comme celles de l'animal lésé. Cet effet est obtenu avec des doses deux fois plus élevées $(2,5 \mu \mathrm{g} / 100 \mathrm{~g})$ que celles utilisées dans l'étude soulignant l'absence de stimulation somatotrope après injection intraveineuse de VIP au rat (Vijayan et al., 1979).

La stimulation de la sécrétion de GH par la TRH ou le VIP semble impliquer une action directe des deux peptides sur la cellule somatotrope puisqu'elle peut être reproduite in vitro aussi bien au cours d'incubation statique que dans un système dynamique de périfusion. Dans ces conditions, la dose efficace 50 (DE50) vis-à-vis de la libération de $\mathrm{GH}$ est de $1,2 \times 10^{-8} \mathrm{~mol} / \mathrm{l}$ pour la $\mathrm{TRH}$ et $2,5 \times 10^{-9} \mathrm{~mol} / \mathrm{l}$ pour le VIP.

Tant pour la TRH que pour le VIP, I'effet sur la sécrétion somatotrope est transitoire, $20 \mathrm{~min}$, malgré le maintien de la périfusion (données non représentées pour le VIPI. Les résultats rapportés dans la littérature concernant l'effet des deux peptides sur la sécrétion de GH apparaissent dépendre de la méthode d'étude et de l'origine de l'hypophyse utilisées. La TRH provoque la sécrétion de GH par des hypophyses de rat périfusées (Carlson, Martitz et Daughaday, 1974) ou par des cellules d'hypophyse bovine en culture (Machlin et al., 1974) mais se révèle sans action sur les cultures de cellules hypophysaires d'origine humaine (Adams, Brajkovich et Mashiter, 1981) ou murine (Szabo et al., 1984) ou au cours d'incubations de demi-hypophyses de rat (Enjalbert et al., 1980). L'effet du VIP sur la sécrétion de $\mathrm{GH}$ a été décrit sur des cellules d'origine tumorale (Aizawa et Hinkle, 1985 ; Chihara et al., 1982, Dorflinger et Schonbrunn, 1983 ; Matsushita et al., 1981 ; White et al., 1985) mais non au cours d'incubations d'hypophyses de rat normal (Enjalbert et al., 1980). L'absence d'effet, dans certaines expériences, de la TRH comme du VIP, peut s'expliquer par l'utilisation de temps d'incu- 
bation trop long par rapport à la brièveté de l'action des peptides que nous observons.

Une action du VIP par l'intermédiaire des récepteurs du GRF, envisageable du fait de l'analogie structurale des deux peptides, paraît peu probable dans nos conditions expérimentales. En effet, si le GRF déplace le VIP de ses récepteurs pancréatiques et intestinaux (Pandol et al., 1984), le VIP jusqu'à une concentration de $10^{-7} \mathrm{~mol} / \mathrm{l}$, ne déplace pas le GRF de ses récepteurs hypophysaires (Seifert et al., 1985).

Plusieurs explications peuvent être apportées à l'absence de stimulation par la TRH de la sécrétion de $\mathrm{GH}$ chez l'animal intact : a) une modification de la réceptivité des hypophyses selon les conditions physiologiques, hypothèse qui peut être écartée puisque, in vitro, nous obtenons la même réponse à la TRH avec l'hypophyse d'animaux lésés ou normaux; b) un hypothyroïdisme consécutif à la lésion qui révélerait un effet de la TRH sur la sécrétion de GH (Chihara et al., 1976b). Cependant, 4 jours après la lésion, la triiodothyroninémie des animaux n'est pas modifiée; c) l'interaction d'un ou plusieurs facteurs hypothalamiques avec la TRH puisque l'effet du tripeptide ne se manifeste qu'après lésion de I'hypothalamus ou in vitro, c'est-à-dire lorsque l'hypophyse ne reçoit plus de messages de l'hypothalamus. II y a, par exemple, perte du rétro-contrôle négatif par la somatostatine.

En conclusion, avec la TRH et le VIP, nous sommes en présence de deux neuropeptides qui exercent directement sur la cellule somatotrope un effet stimulant à des concentrations compatibles avec une intervention physiologique de ces peptides sur la sécrétion de l'hormone de croissance. II reste à déterminer le ou les facteurs qui interfèrent de façon négative avec la TRH.

$12^{e}$ Réunion du groupe Développement I.N.R.A., Montpellier, 28-30 mai 1986.

\section{Références}

ADAMS E. F., BRAJKOVICH I. E., MASHITER K., 1981. Growth hormone and prolactin secretion by dispersed cell cultures of a normal human pituitary : effects of thyrotrophin releasing hormone, theophylline, somatostatin and 2 bromo- $\alpha$-ergocryptine. Acta endocrin., Copenh., 98 , 345-351.

AIZAWA T., HINKLE P. M., 1985. Differential effects of thyrotropin releasing hormone, vasoactive intestinal peptide, phorbol ester and depolarization in $\mathrm{GH}_{4} \mathrm{C}_{1}$ rat pituitary cells. Endocrinology, 116, 909-919.

BLUET-PAJOT M. T., DURAND D., DROUVA S. V., MOUNIER F., PRESSAC M., KORDON C., 1986. Further evidence that thyrotropin-releasing hormone participates in the regulation of growth hormone secretion in the rat. Neuroendocrinology, (sous presse).

BORGES J. L. C., USKAVITCH D. R., KAISER D. L., CRONIN M. J., EVANS W. S., THORNER M. O., 1983. Human pancreatic growth hormone-releasing factor-40 (hp GRF-40) allows stimulation of $\mathrm{GH}$ release by TRH. Endocrinology, 113, 1519-1521.

BRAZEAU P., VALE W., BURGUS R., LING N., BUTCHER M., RIVIER J., GUILLEMIN R., 1973. Hypothalamic polypeptide that inhibits the secretion of immunoreactive pituitary growth hormone. Science, 179, 77-79.

BROWN M., VALE W., 1975. Growth hormone release in the rat: effects of somatostatin and thyrotropin releasing factor. Endocrinology, 97, 1151-1156. 
CARLSON H. E., MARITZ I. K., DAUGHADAY W. H., 1974. Thyrotropin releasing hormone stimulation and somatostatin inhibition of growth hormone secretion from perfused rat adenohypophyses. Endocrinology, 94, 1709-1713.

CHIHARA K., KATO Y., OHGO S., IWASAKI Y., ABE H., MAEDA K., IMURA H., 1976a. Stimulating and inhibiting effects of thyrotropin releasing hormone on growth hormone release in rats. Endocrinology, 98, 1047-1053.

CHIHARA K., KATO Y., OHGO S., IWASAKI Y., MAEDA K., 1976b. Effects of hyperthyroidism and hypothyroidism on rat growth hormone release induced by thyrotropin-releasing hormone. Endocrinology, 98, 1396-1400.

CHIHARA K., IWASAKI J., MINAMITANI N., KAJI M., MATSUKURA S., TAMAKI N., MATSUMOTO S., FUJITA T., 1982. Effect of vasoactive intestinal polypeptide on growth hormone secretion in perifused acromegalic pituitary adenoma tissues. J. clin. Endocrinol. Metab., 54, 773-779.

DENEF C., SCHRAMME C., BAES M., 1985. Stimulation of growth hormone release by vasoactive intestinal peptide and peptide $\mathrm{PHI}$ in rat anterior pituitary reaggregates. Neuroendocrinology, 40, 88-91.

DORFLINGER L. J., SCHONBRUNN A., 1983. Somatostatin inhibits vasoactive intestinal peptide stimulated cyclic adenosine monophosphate accumulation in GH pituitary cells. Endocrinology, 113, 1551-1558.

DUNN J., ARIMURA A., 1974. Serum growth hormone levels following ablation of mediobasal hypothalamus. Neuroendocrinology, 15, 189-199.

ENJALBERT A., ARANCIBIA S., RUBERG M., PRIAM M., BLUET-PAJOT M. T., ROTSZTEJN W. H., KORDON C., 1980. Stimulation of in vitro prolactin release by vasoactive intestinal peptide. Neuroendocrinology, 31, 200-204.

GUILLEMIN R., BRAZEAU P., BOHLEN P., ESCH F., LING N., WEHRENBERG W. B., 1982. Growth hormone releasing factor from a human pancreatic tumor that causes acromegaly. Science, 218, 585-587.

IRIE M., TSUSHIMA T., 1972. Increase of serum growth hormone concentration following thyrotropin releasing hormone injection in patients with acromegaly or gigantism. J. clin. Endocr. Metab., 35, 97-100.

KATO Y., CHIHARA K., MAEDA K., OHGO S., OKANISHI Y., IMURA H., 1975. Plasma growth hormone responses to thyrotropin releasing hormone in the urethane anesthetized rat. Endocrinology, 96, 1114-1118.

MACHLIN L. J., JACOBS L. S., CIRULIS N., KIMES R., MILLER R., 1974. An assay for growth hormone and prolactin releasing activities using a bovine pituitary cell culture system. Endocrinology, 95, 1350-1358.

MARTIN J. B., 1973-1974. Studies on the mechanism of pentobarbital induced GH release in the rat. Neuroendocrinology, 13, 339.

MATSUSHITA N., KATO Y., KATAKAMI H., SHIMATSU A., YANAIHARA N., IMURA H., 1981. Stimulation of growth hormone release by vasoactive intestinal polypeptide from human pituitary adenomas in vitro. J. clin. Endocrinol. Metab., 53, 1297-1300.

MÜLLER E. E., PANERAI A. E., COCCHI D., GIL-AD I., ROSSI G. L., OLGIATI V. R., 1977. Growth hormone releasing activity of thyrotropin releasing hormone in rats with hypothalamic lesions. Endocrinology, 100, 1663-1677.

PANDOL S. J., SEIFERT H., THOMAS M. W., RIVIER J., VALE W., 1984. Growth hormonereleasing factor stimulates pancreatic enzyme secretion. Science, 225, 326-328.

PARKER R. B., WAUD D. R., 1971. Pharmacological estimation of drug receptor dissociation constants. I : Agonists. J. Pharmac. exp. Ther., 177, 1-12.

ROTSZTEJN W. H., BENOIST L., BESSON J., BERAUD G., BLUET-PAJOT M. T., KORDON C., ROSSELIN G., DUVAL J., 1980. Effect of vasoactive intestinal peptide (VIP) on the release of adenohypophyseal hormones from purified cells obtained by unit gravity sedimentation. Neuroendocrinology, 31, 282-286.

SEIFERT H., PERRIN M., RIVIER J., VALE W., 1985. Binding sites for growth hormone releasing factor on rat anterior pituitary cells. Nature, 313, 487-489.

SZABO M., STACHURA M. E., PALEOLOgOS N., BYBEE D. E., FROHMAN L. A., 1984. Thyrotropin releasing hormone stimulates growth hormone release from the anterior pituitary of hypothyroid rats in vitro. Endocrinology, 114, 1344-1351. 
TACHÉ Y., DU RUISSEAU P., DUCHARME J. R., COLLU R., 1977. A.ntagonism of pentobarbital induced hormonal changes by TRH in rats. Eur. J. Pharmacol., 45, 369-376.

VIGH S., SCHALLY A. V., 1984. Interaction between hypothalamic peptides in a superfused pituitary cell system. Peptides, 5, 241-247.

VIJAYAN E., SAMSON W. K., SAID S. I., MCCANN S. M., 1979. Vasoactive intestinal peptide. Evidence for a hypothalamic site of action to release growth hormone, luteinizing hormone and prolactin in conscious ovariectomized rats. Endocrinology, 104, 53-57.

WHITE M. C., DANIELS M., KENDALL-TAYLOR P., TURNER S. J., MATHIAS D., TEASDALE G., 1985. Effects of growth hormone releasing factor (1-44) on growth hormone release from human somatotrophinomas in vitro: interaction with somatostatin, dopamine, vasoactive intestinal peptide and cycloheximide. J. Endocr., 105, 269-276. 